\title{
Gobierno de las TI en las universidades: análisis sistemático de la literatura científica y no convencional
}

\section{Government of the TI in the universities: systematic analysis of the scientific and unconventional literature}

Francisco Valverde-Alulema

Robert Enríquez-Reyes

Universidad Central del Ecuador, Ecuador

Faraón Llorens-Largo

Universidad de Alicante, España

Autor para correspondencia: fvalverde@uce.edu.ec; renriquez@uce.edu.ec;

Faraon.Llorens@ua.es

Fecha de recepción: 07 de Agosto de 2017 - Fecha de aceptación: 10 de agosto de 2017

Resumen: El trabajo de investigación propone una revisión sistemática de la literatura científica y la literatura no convencional, con el fin de identificar exhaustivamente los estudios pertinentes a la pregunta de investigación ¿existen investigaciones en el ámbito del Gobierno de TI en las Universidades? Se ha consultado WOS, SCOPUS y Google Académico y bases de datos no convencionales de universidades y asociaciones internacionales. Las palabras claves de búsqueda han sido gobernanza de las TI y universidades. Los criterios de inclusión han sido publicaciones escritas en español o inglés y entre los años 2000 y 2017. Se ha realizado una búsqueda de artículos, publicaciones de conferencias y libros en distintas bases de datos científicas. En la literatura no convencional, se ha tenido en cuenta tesis, informes, artículos, reportes y normas internacionales, de repositorios de Universidades y de Asociaciones internacionales. Las áreas del conocimiento han sido Ciencias de la Computación, Ingeniería, Administración y Negocios, Economía y Finanzas, Ciencias de Decisión y Tecnologías de la Información y Comunicación. Se han encontrado un total de 386 publicaciones relacionadas con el gobierno de la TI en universidades, de las cuales 316 están en bases de datos científicas y 70 publicaciones recopiladas de bases de datos no convencionales. Los resultados obtenidos demuestran el creciente interés de la comunidad científica en el tema, donde la mayor parte de publicaciones pertenecen a Ciencias de la Computación, lo que justifica la línea de investigación de Gobernanza de las TI y en estudios específicos para Universidades donde se debe continuar investigando.

Palabras clave: gobierno de ti; universidades; slr; literatura no convencional

Abstract: The research work proposes a systematic review of scientific literature and nonconventional literature, in order to exhaustively identify the relevant studies to the research question are there research in the field of IT Governance in Universities? WOS, SCOPUS and Google Scholar have been consulted and unconventional databases of universities and international associations have been consulted. Search keywords have been governance of IT and universities. The inclusion criteria have been publications written in Spanish or English and between 2000 and 2017. A search for articles, conference publications and books has been carried out in different scientific databases. In the non-conventional literature, it has taken into account 
theses, reports, articles, reports and international standards, of repositories of Universities and International Associations. The areas of knowledge have been Computer Science, Engineering, Administration and Business, Economics and Finance, Decision Sciences and Information and Communication Technologies. A total of 386 publications related to IT governance have been found in universities, of which 316 are in scientific databases and 70 publications compiled from non-conventional databases. The results obtained demonstrate the growing interest of the scientific community in the subject, where most publications belong to Computer Science, which justifies the research line of IT Governance and specific studies for Universities where it is necessary to continue doing research.

Key words: it government; universities; slr; non-conventional literature

\section{Introducción}

\section{Gobierno Corporativo de las TI en Universidades}

El Gobierno corporativo de las Tecnologías de la Información (TI) es el sistema más adecuado para que una organización alcance la máxima eficiencia en la gestión de sus TI. Pero, para tener éxito debe basarse en un buen Gobierno Corporativo, que establezca claramente los objetivos de negocio e incluya la planificación estratégica de las TI como herramienta para alinear los objetivos de negocio con los objetivos de las TI. (Fernández, 2009).

Las TI tienen un carácter estratégico transversal, apoyan al cumplimiento de objetivos organizacionales, por tanto, deben formar parte de la planificación global de la universidad. Las responsabilidades relacionadas con la planificación de TI deben recaer y ser apoyadas por la más alta dirección universitaria. Sin el apoyo de esta y su concienciación al incluir a las TI como parte fundamental de la institución, se ocasionará un retraso organizacional y, por ende, este tipo de universidades estarán sentenciadas a desaparecer en el corto y mediano plazo. (Fernandez \& Llorens, 2011)

Según afirma Uceda Antolín, Barro Ameneiro, Llorens Largo, y Franco Tubio (2010) "Las TI no deberían ser un fin en sí mismo, sino un medio que contribuya a crear valor a las universidades" (p.105). Si las universidades no consiguen que sus TI generen valor, estas serán ineficientes y perderán una gran ventaja competitiva.

Para lograr mantener esta ventaja competitiva se han puesto en marcha diversos proyectos internacionales, tal como el proyecto GTI4U (Gobierno de las TI para Universidades). Tanto el modelo como la metodología utilizada son adecuados para establecer sistemas de Gobierno de las TI en Universidades, no sólo de España (Fernández Martínez y Llorens Largo, 2014, p.91), sino también se empieza a trabajar en Latinoamérica. Tras diez años de evaluación comparativa en España, se empezó a implementar la aplicación de las mismas herramientas en América Latina (Fernández Martínez, Llorens Largo, y Hontoria Hernández, 2015).

Por otro lado, se observa que, para el uso efectivo de las TI, varias organizaciones internacionales han publicado una serie de documentos que permiten la aplicación de gobierno de las TI. Los documentos claves son COBIT, ISO 38500, ISO 20000 e ITIL (Bin-Abbas \& Bakry, 2014, p261). Estos documentos han sido muy difundidos en los ámbitos de las 
Direcciones de TI, especialmente en el área financiera donde existen regulaciones para la implementación por parte de los gobiernos.

Con todo lo anteriormente descrito, queda claro el interés de las organizaciones, y en particular de las universidades, por aplicar e implementar una cultura de Gobernanza de las TI. Pero ¿existe el mismo interés en la comunidad científica por investigar sobre el tema? Para poder responder a esta pregunta se ha realizado una revisión sistemática de la literatura científica (SLR - Systematic Literature Review) existente sobre Gobierno TI en Universidades. Se han tenido en cuenta publicaciones no convencionales relacionadas al tema de repositorios de universidades y de asociaciones de alto prestigio, tales como tesis doctorales, tesis de maestría, informes y marcos de referencia, entre otros.

\section{Revisión Sistemática de la Literatura}

Según afirma Sánchez-Meca (2010), las revisiones sistemáticas son un tipo de investigación científica que tiene como propósito integrar de forma objetiva y sistemática los resultados de los estudios empíricos sobre un determinado problema de investigación, con objeto de determinar el estado de la cuestión en ese campo de estudio.

Un análisis sistemático tiene como objetivo identificar exhaustivamente todos los estudios pertinentes para responder a una pregunta en particular, y evalúa la validez, o solidez, de cada estudio teniendo esto en cuenta al extraer conclusiones (Ferreras, 2016).

En su trabajo Ferreras deduce que "la revisión sistemática deberá cumplir al menos tres condiciones:

1. Utilizar bases de datos académicas como fuente principal.

2. Indicar los criterios de inclusión (y si es el caso, de exclusión) para seleccionar las obras que han formado parte del corpus de análisis.

3. Proporcionar datos para replicar el estudio de revisión." (2016)

Así, nuestra hipótesis a demostrar es: ¿existen investigaciones en el ámbito del Gobierno de TI en la Universidades? Esta revisión sistemática (SLR) de literatura científica y no convencional es necesaria para determinar si se está trabajando en Gobierno de TI en las Universidades, en qué bases científicas se encuentran publicados estos trabajos y cuántos trabajos existen. Los resultados de la revisión servirán para aportar en nuevas investigaciones sobre temas puntuales del Gobierno de TI y como mejorar la administración de la TI en las Universidades.

Para la revisión se ha utilizado las bases de datos Web of Science (WOS) y SCOPUS contando con un equipo de investigación multidisciplinario de diferentes Facultades de la Universidad Central del Ecuador y de la Universidad de Alicante de España.

\section{Revisión de la literatura no convencional}

La literatura no convencional, según se afirma en el Sixth International Conference on Grey Literature (1997), “es aquella que se produce a instancias del gobierno, de la enseñanza e 
investigación pública, del comercio y de la industria en formato impreso o electrónico, pero que no está controlada por editores comerciales". Pero esto no implica que no tengan revisiones de pares que las hacen útiles para los estudios científicos. Aunque la pregunta que nos planteamos es si existen investigaciones sobre el gobierno de las TI en universidades, por la propia naturaleza del tema no podemos obviar las fuentes procedentes de asociaciones profesionales. Además, en los tiempos actuales, cada vez está más accesible la literatura científica no convencional (trabajos de tesis, actas de congresos...) gracias a las tecnologías digitales. Así, la búsqueda en las bases de datos no convencionales de universidades y de asociaciones internacionales es también parte importante de esta investigación.

\section{Materiales y métodos}

La metodología para desarrollar una revisión sistemática (SLR), propone la utilización de palabras clave para delimitar la búsqueda. En este caso se ha utilizado dos términos "Gobernanza de las TI" y "Universidades", con sus distintas variantes terminológicas (gobierno de las TI, IT governance).

Las bases de datos científicas consultadas han sido WOS, Scopus y Google Académico. Además, por la particularidad del tema de investigación, se complementa el estudio con las bases de datos no convencionales de universidades y de asociaciones internacionales reconocidas y de alto prestigio.

Los criterios de inclusión aplicados han sido:

- Idioma: español e inglés

- Años: desde el año 2000 hasta la actualidad (2017).

- Tipo de publicaciones. En las bases de datos científicas: artículos, papers de conferencias y libros. En la literatura no convencional: tesis, informes, artículos, reportes y normas internacionales.

- Áreas del conocimiento: Ciencias de la Computación, Ingeniería, Administración y Negocios, Economía y Finanzas, Ciencias de Decisión y Tecnologías de la Información y Comunicación.

Para lograr los objetivos del estudio, se ha utilizado la metodología de Revisión Sistemática de la Literatura (SLR), la misma apoyará con la organización de las publicaciones de interés y relevancia, para la investigación del problema propuesto. El método permite utilizar pasos debidamente ordenados secuencialmente, organizar las publicaciones halladas en bases de datos científicas y en bases de datos no convencionales, y posteriormente, con un análisis estadístico, establecer parámetros de aportación y relevancia de las publicaciones, de este modo se justifica y valida la investigación realizada.

De acuerdo a la metodología, una vez escogidos los dos términos clave, se ha realizado un análisis de todas las palabras semejantes, conectándolas con el conector lógico OR al realizar la pregunta de búsqueda. Para Gobernanza de TI se han tenido en cuenta los siguientes términos: 
"Gobierno de TI" OR "Gobernanza de TI" OR "IT Government" OR "IT Governance" OR "Gobierno de Tecnologías" OR "Gobernanza de Tecnologías" OR "Technology Government" OR "Technology Governance" OR "Technologies Government" OR "Technologies Governance" OR "Gobierno de tecnologías de la información" OR "Gobernanza de tecnologías de la información" OR "Information Technology Governmente" OR "Information Technology Governance" OR "Information Technologies Government" OR "Information Technologies Governance" OR "Gobierno Corporativo de TI" OR "Gobernanza Corporativa de TI" OR "Governance of IT" OR "Government of IT" OR "Governance of Technologies" OR "Governance of Technology".

Y para Universidades:

"Instituciones" OR "Universidades" OR "Universidad" OR "Educación Superior" OR "Instituciones Superiores" OR "Institución Superior" OR "High Education" OR "University" OR "Universities" OR "Institution" OR "Institutions" OR "Education" OR "Education of University" OR "Education of Universities"

Entre las dos palabras clave se ejecuta el conector lógico AND y con estos datos se realiza la búsqueda en las bases de datos científicas y no convencionales. Las palabras deben estar entre comillas para denotar que la búsqueda sea específica de las mismas y dentro del título de la publicación y palabras clave.

Una vez realizada la búsqueda los resultados se ingresan en una hoja de cálculo con los campos de: base de datos científica, tipo de documento, revista científica o congreso, idioma, área de conocimiento, año de publicación, número de citas, autores, título. Con esta base de información se realiza el análisis estadístico para encontrar los resultados que nos permitan responder a nuestra hipótesis de investigación.

\section{Resultados}

Tras la recolección de documentos se han encontrado 386 publicaciones. En la tabla 1 se presenta un resumen del resultado de búsquedas de las publicaciones relacionadas. Se observa que 316 publicaciones proceden de bases de datos científicas y 70 publicaciones de bases de datos no convencionales que complementan la investigación. Se ha realizado una depuración de las publicaciones eliminándose aquellas que aparecían repetidas.

\section{Tabla 1. Resultados de refinamientos de búsquedas de las publicaciones relacionadas a la investigación}




\begin{tabular}{|c|c|c|c|}
\hline \multicolumn{4}{|c|}{ PUBLICACIONES RELACIONADAS } \\
\hline \multicolumn{3}{|c|}{ BASES DE DATOS CIENTÍFICAS } & PORCENTAJE \\
\hline TOTAL PUBLICACIONES DE WOS & 82 & PORCENTAJE WOS & 25,95 \\
\hline TOTAL PUBLICACIONES SCOPUS & 202 & PORCENTAJE SCOPUS & 63,92 \\
\hline $\begin{array}{l}\text { TOTAL PUBLICACIONES GOOGLE } \\
\text { SCHOOLAR }\end{array}$ & 32 & $\begin{array}{l}\text { PORCENTAJE GOOGLE } \\
\text { SCHOOLAR }\end{array}$ & 10,13 \\
\hline TOTAL BDC & 316 & & 100 \\
\hline \multicolumn{3}{|c|}{ LITERATURA GRIS (NO CONVENCIONAL O SEMIPUBLICADA) } & PORCENTAUE \\
\hline PUBLICACIONES UNIVERSIDADES & 20 & $\begin{array}{l}\text { PORCENTAJE } \\
\text { UNIVERSIDADES }\end{array}$ & 28,57 \\
\hline PUBLICACIONES ASOCIACIONES & 50 & $\begin{array}{l}\text { PORCENTAJE } \\
\text { ASOCIACIONES }\end{array}$ & 71,43 \\
\hline TOTAL LG & 70 & & 100 \\
\hline
\end{tabular}

En la tabla 2 se observa la distribución de las 386 publicaciones por área de conocimiento. Se han encontrado 158 del Área de Ciencias de la Computación, 57 de Ingeniería, 67 en Administración y Negocios, 14 en Economía y Finanzas, 26 de Ciencias de la Decisión y 64 de Tecnologías de la Información. La figura 1 presenta un diagrama de las publicaciones de acuerdo al área de conocimiento donde se destaca claramente el área de Ciencias de la Computación (con 158). También observamos que hay un número considerable de publicaciones en las áreas de Administración y Negocios (67), de Tecnologías de la Información (64) y de Ingeniería (57).

Tabla 2. Resultados por Área de Conocimiento de las publicaciones relacionadas a la investigación

\begin{tabular}{|c|c|c|c|c|}
\hline \multirow[b]{2}{*}{ AREA DEL CONOCIMIENTO } & \multirow[b]{2}{*}{$\begin{array}{c}\text { BASE DE } \\
\text { DATOS CIENT. }\end{array}$} & \multicolumn{2}{|c|}{ LITERATURA GRIS } & \multirow[b]{2}{*}{ TOTAL } \\
\hline & & $\begin{array}{l}\text { LITERATURA GRIS } \\
\text { UNIVERSIDADES }\end{array}$ & $\begin{array}{c}\text { LITERATURA GRIS } \\
\text { ASOCIACIONES }\end{array}$ & \\
\hline CIENCIAS DE LA COMPUTACIÓN & 154 & 4 & 0 & 158 \\
\hline INGENIERÍA & 51 & 4 & 2 & 57 \\
\hline ADMINISTRACIÓN Y NEGOCIOS & 61 & 3 & 3 & 67 \\
\hline ECONOMÍA Y FINANZAS & 11 & 3 & 0 & 14 \\
\hline CIENCIAS DE DECISIÓN & 26 & 0 & 0 & 26 \\
\hline TECNOLOGÍAS DE LA INFORMACIÓN & 13 & 6 & 45 & 64 \\
\hline TOTAL & 316 & 20 & 50 & 386 \\
\hline
\end{tabular}




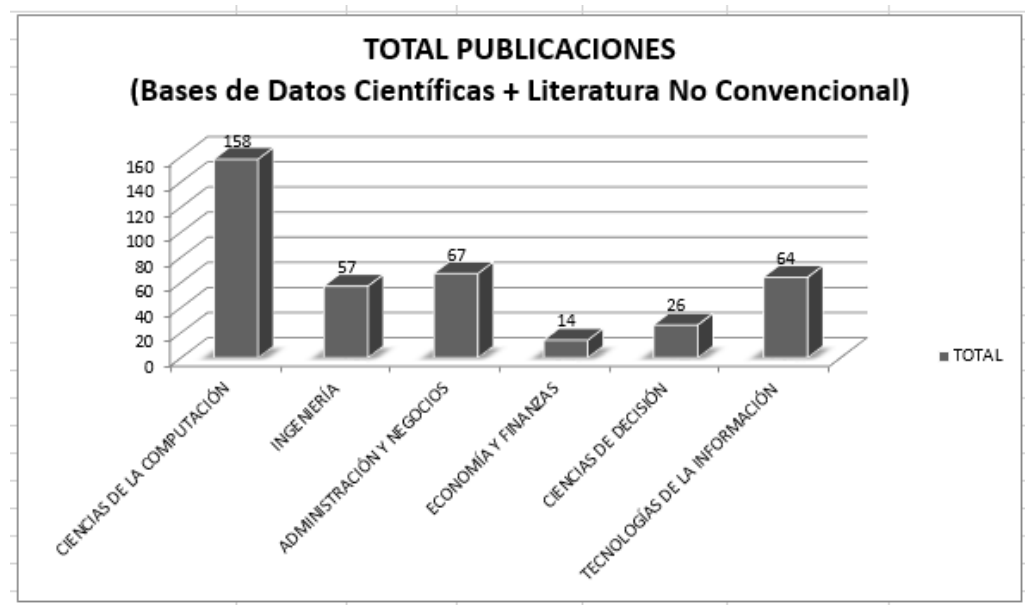

Figura 1. Gráfica de publicaciones totales por área de conocimiento incluidas bases de datos científicas y publicaciones de literatura no convencional.

Al analizar las publicaciones, separándolas por las bases de datos científicas y la literatura no convencional, se destaca el área de Ciencias de la Computación con 154 publicaciones como se puede ver en la figura 2. También observamos el área de Administración y Negocios con 61 publicaciones, seguida por el área de Ingeniería con 51 publicaciones. En la figura 3 se muestra las publicacones de la literatura no convencional, las publicaciones que se destacan son las referentes al área de Tecnologías de la Información (6), con mayor número de publicaciones, seguida por el área de Ciencias de la Computación (4) e Ingeniería (4). En repositorios de asociaciones internacionales, las publicaciones que se destacan en su gran mayoría son del área de las Tecnologías de la Información (figura 4).

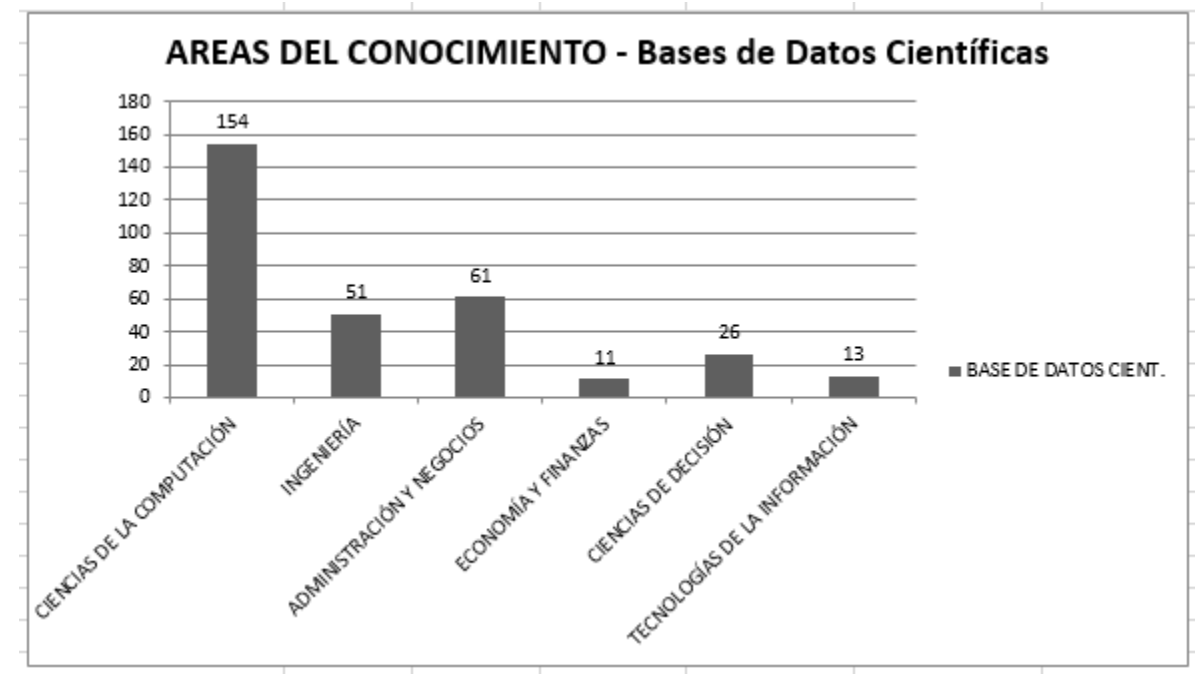

Figura 2. Gráfica de publicaciones por área de conocimiento en Bases de Datos Científicas. 


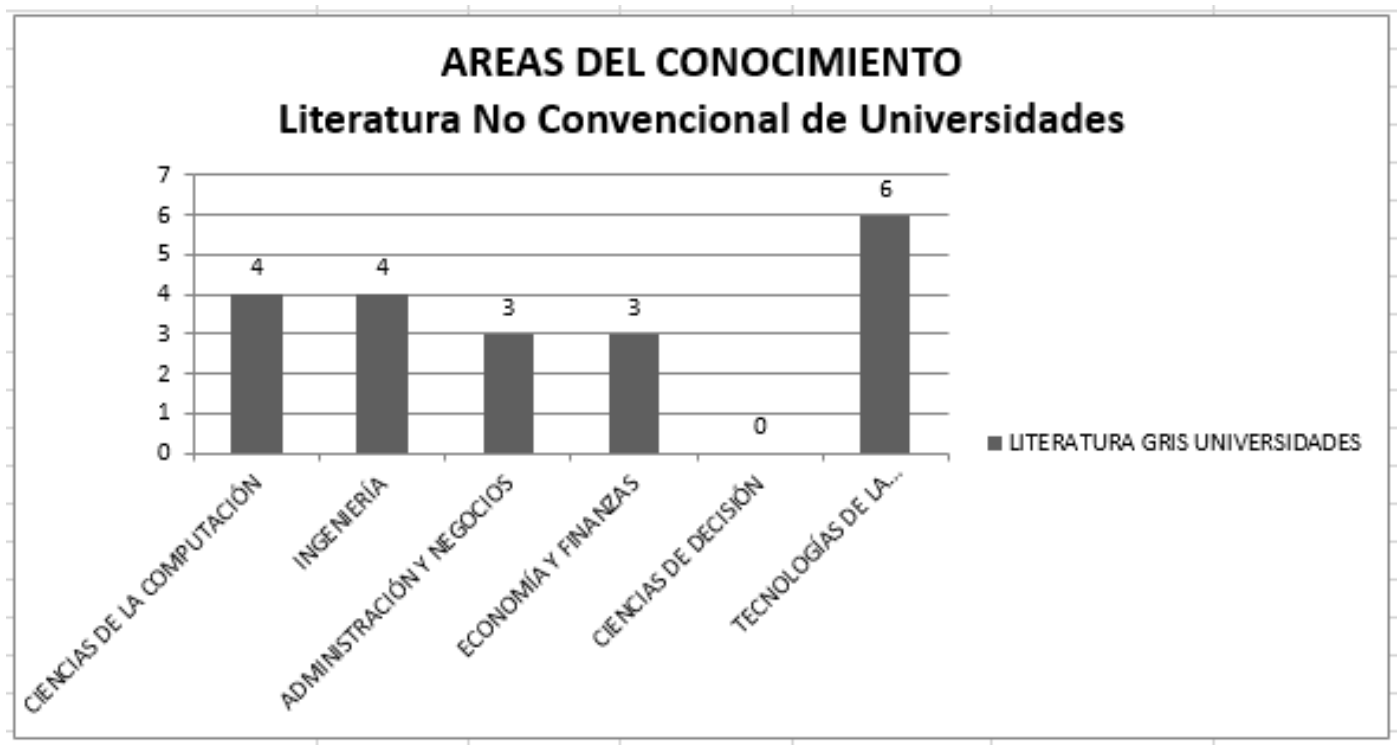

Figura 3. Gráfica de publicaciones por área de conocimiento en repositorios de literatura no convencional de Universidades.

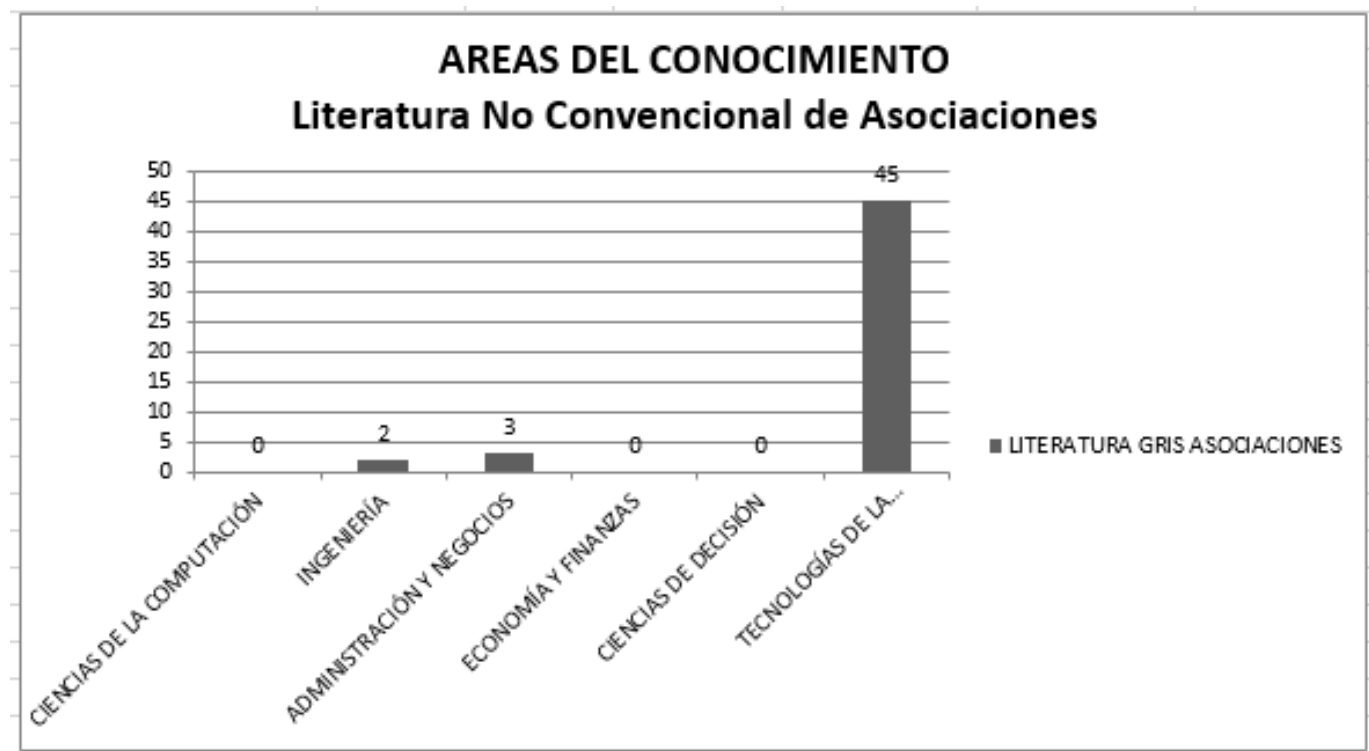

Figura 4. Gráfica de publicaciones por área de conocimiento en repositorios de literatura no convencional de Asociaciones internacionales de prestigio.

La tabla 3 presenta en detalle la fecha de publicación de los distintos trabajos encontrados, separados por tipo de bases de datos de procedencia, la evolución temporal se grafica en la figura 5. El número obtenido para el año 2017 no es comparable con los de los demás años, ya que la búsqueda se ha realizado al finalizar el primer trimestre del año. La evolución desde el año 2000 ha sido constante y ascendente, obteniendo su valor máximo en el año 2014 con 53 publicaciones. Estos datos constatan el creciente interés de la comunidad científica por el tema del gobierno de las TI en las universidades.

Tabla 3. Resultados por año de las publicaciones relacionadas a la investigación 


\begin{tabular}{|c|c|c|c|c|}
\hline AÑO & $\begin{array}{c}\text { BASES DE } \\
\text { DATOS } \\
\text { CIENTÍFICAS }\end{array}$ & $\begin{array}{c}\text { LITERATURA NO } \\
\text { CONVENCIONAL } \\
\text { UNIVERSIDADES }\end{array}$ & $\begin{array}{c}\text { IITERATURA NO } \\
\text { CONVENCIONAL } \\
\text { ASOCIACIONES }\end{array}$ & TOTAL \\
\hline AÑO 2000 & 0 & 0 & 3 & 3 \\
\hline AÑO 2001 & 3 & 0 & 0 & 3 \\
\hline AÑ̃ 2002 & 1 & 0 & 0 & 1 \\
\hline AÑO 2003 & 5 & 0 & 2 & 7 \\
\hline AÑO 2004 & 10 & 2 & 4 & 16 \\
\hline AÑO 2005 & 7 & 1 & 1 & 9 \\
\hline AÑO 2006 & 17 & 0 & 1 & 18 \\
\hline AÑO 2007 & 19 & 2 & 1 & 22 \\
\hline AÑO 2008 & 19 & 0 & 5 & 24 \\
\hline AÑ̃ 2009 & 25 & 2 & 6 & 33 \\
\hline AÑ̃ 2010 & 18 & 0 & 2 & 20 \\
\hline AÑO 2011 & 25 & 0 & 2 & 27 \\
\hline AÑ̃ 2012 & 21 & 1 & 7 & 29 \\
\hline AÑ̃ 2013 & 32 & 5 & 2 & 39 \\
\hline AÑO 2014 & 46 & 3 & 4 & 53 \\
\hline AÑO 2015 & 38 & 2 & 7 & 47 \\
\hline AÑ̃ 2016 & 23 & 2 & 3 & 28 \\
\hline AÑ̃ 2017 & 7 & 0 & 0 & 7 \\
\hline & 316 & 20 & 50 & 386 \\
\hline
\end{tabular}

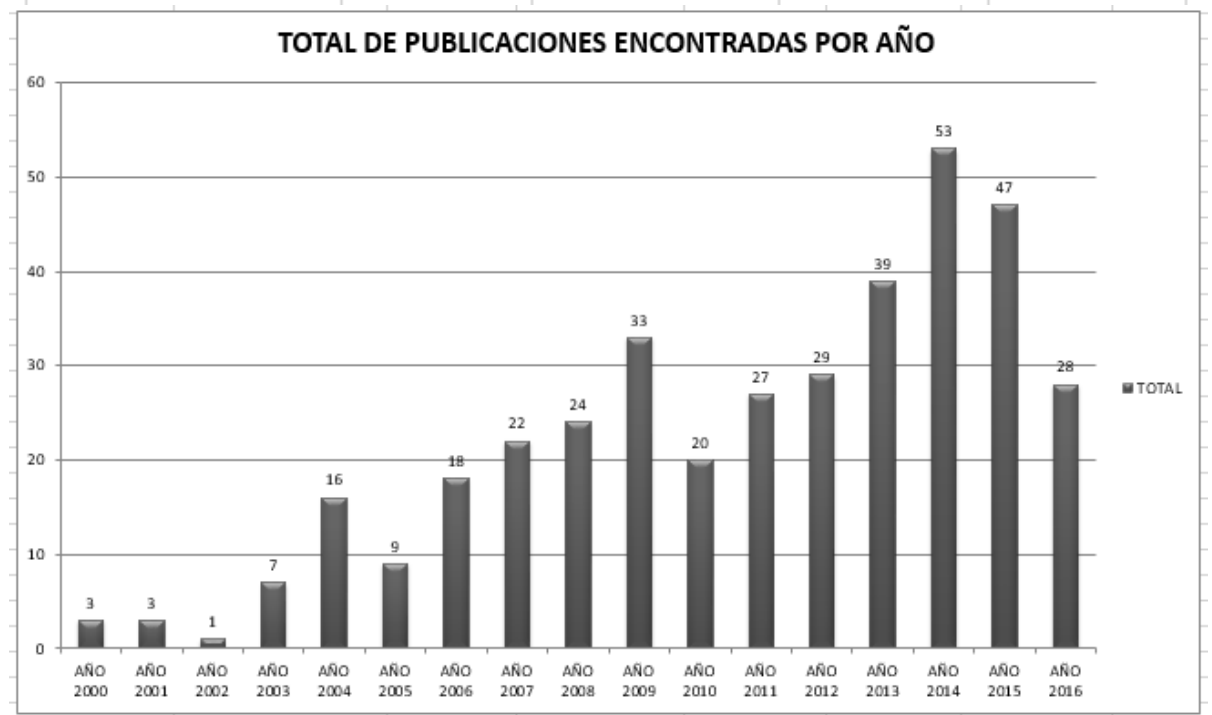

Figura 5. Gráfica de publicaciones por año.

Las publicaciones, teniendo en cuenta el idioma en el que están escritas (tabla 4 y figura 6), de un total de 386 publicaciones, 341 están escritas en inglés $(88,34 \%$ ) y 45 en español $(11,66 \%)$, Las publicaciones en inglés son significativamente mayores a las de español.

Tabla 4. Resultados por idioma de las publicaciones relacionadas a la investigación

\begin{tabular}{|l|c|c|c|c|}
\hline IDIOMA & $\begin{array}{c}\text { BASES DE } \\
\text { DATOS } \\
\text { CIENTÍFICAS }\end{array}$ & $\begin{array}{c}\text { LITERATURA NO } \\
\text { CONVENCIONAL } \\
\text { UNIVERSIDADES }\end{array}$ & $\begin{array}{c}\text { UTTERATURA NO } \\
\text { CONVENCIONAL } \\
\text { ASOCIACIONES }\end{array}$ & TOTAL \\
\hline INGLES & 310 & 5 & 26 & 341 \\
\hline ESPAÑOL & 6 & 15 & 24 & 45 \\
\hline TOTAL & 316 & 20 & 50 & 386 \\
\hline
\end{tabular}




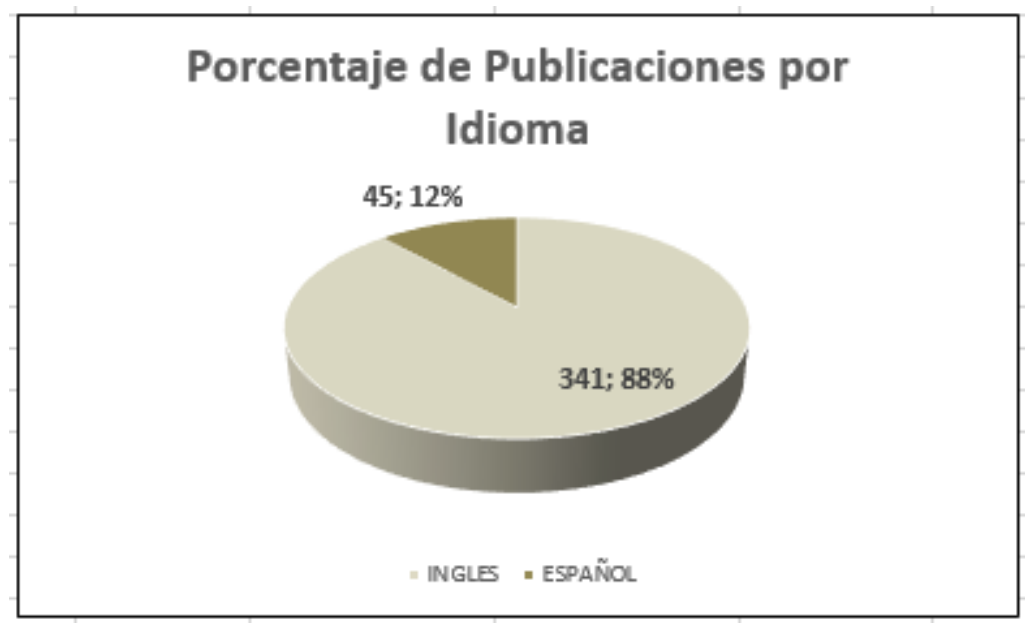

Figura 6. Gráfica de publicaciones por idioma

De las 386 publicaciones (tabla 5 y figura 7), se observa que existe un mayor número en artículos de investigación (189), seguidos de papers de conferencia (147). Existe una considerable contribución de informes (21) y libros (18) relacionados al ámbito de estudio. Las bases de datos científicas han aportado mayoritariamente artículos, en cambio la literatura no convencional, libros e informes. Es importante tener en cuenta las tesis doctorales (3) y de maestría (5), no tanto por su cantidad si no por su interés para el presente trabajo de investigación.

Tabla 5. Resultados totales por tipo de documento de las publicaciones relacionadas a la investigación

\begin{tabular}{|l|c|c|c|c|}
\hline $\begin{array}{c}\text { TIPO DE } \\
\text { DOCUMENTO }\end{array}$ & $\begin{array}{c}\text { BASES DE } \\
\text { DATOS } \\
\text { CIENTÍFICAS }\end{array}$ & $\begin{array}{c}\text { LITERATURA NO } \\
\text { CONVENCIONAL } \\
\text { UNIVERSIDADES }\end{array}$ & $\begin{array}{c}\text { LITERATURA NO } \\
\text { CONVENCIONAL } \\
\text { ASOCIACIONES }\end{array}$ & TOTAL \\
\hline ARTÍCULO & 164 & 6 & 19 & 189 \\
\hline $\begin{array}{l}\text { PAPER DE } \\
\text { CONFERENCIA }\end{array}$ & 144 & 1 & 2 & 147 \\
\hline LIBRO & 8 & 0 & 10 & 18 \\
\hline INFORME & 0 & 5 & 16 & 21 \\
\hline NORMA & 0 & 0 & 3 & 3 \\
\hline $\begin{array}{l}\text { TESIS } \\
\text { DOCTORAL }\end{array}$ & 0 & 3 & 0 & 3 \\
\hline $\begin{array}{l}\text { TESIS } \\
\text { MAESTRÍA }\end{array}$ & 0 & 5 & 0 & 5 \\
\hline \multicolumn{1}{|c|}{ TOTAL } & 316 & 20 & 50 & 386 \\
\hline
\end{tabular}

De las 316 publicaciones encontradas en las bases de datos científicas, 144 son publicaciones de artículos presentados en congresos internacionales. La tabla 6 muestra el top 10 de los congresos más referenciadas. Se observa que los congresos en donde se han publicado más artículos referentes al tema de estudio son los organizados por IEEE con 22 publicaciones. 

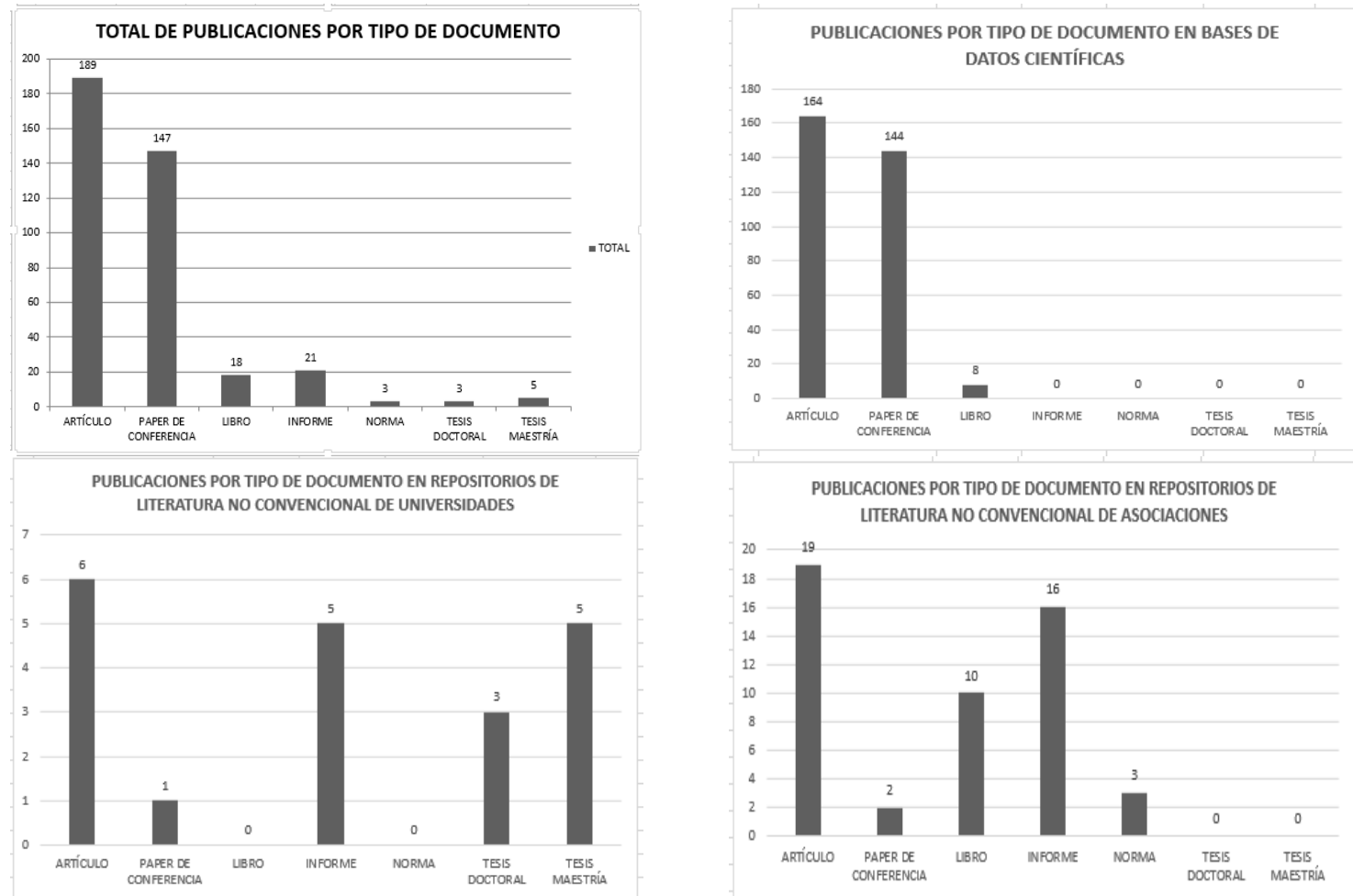

Figura 7. Gráfica de publicaciones por tipo de documento

Tabla 6. Congresos Internacionales más referenciados respecto a Gobierno de TI en Universidades

\begin{tabular}{|c|c|c|}
\hline \multicolumn{3}{|c|}{$\begin{array}{l}\text { CONGRESOS INTERNACIONALES MAS REFERENCIADOS EN BASES DE DATO } \\
\text { CIENTÍFICAS }\end{array}$} \\
\hline Nro. & CONGRESOS & REFERENCIADOS \\
\hline 1 & IEEE & 22 \\
\hline 2 & $\begin{array}{l}\text { Hawaii International Conference on } \\
\text { System Sciences, HICSS }\end{array}$ & 10 \\
\hline 3 & $\begin{array}{l}\text { International Business-Information- } \\
\text { Management-Association Conference, }\end{array}$ & 9 \\
\hline 4 & $\begin{array}{l}\text { European Conference on Information } \\
\text { Systems, ECIS }\end{array}$ & 8 \\
\hline 5 & $\begin{array}{l}\text { Americas Conference on Information } \\
\text { Systems, AMCIS }\end{array}$ & 7 \\
\hline 6 & $\begin{array}{l}\text { Australasian Conference on Information } \\
\text { Systems, ACIS }\end{array}$ & 7 \\
\hline 7 & $\begin{array}{l}\text { International Conference on Engineering } \\
\text { and Technology, (ICET) }\end{array}$ & 6 \\
\hline 8 & $\begin{array}{l}\text { International Conference on Information } \\
\text { Security and Assurance, ISA }\end{array}$ & 6 \\
\hline 9 & $\begin{array}{l}\text { International Conference on Information } \\
\text { and Communication Technology for the } \\
\text { Muslim World, ICT4M }\end{array}$ & 5 \\
\hline 10 & $\begin{array}{l}\text { International Conference on } \\
\text { InformationScience and Security, ICISS }\end{array}$ & 5 \\
\hline & TOTAL & 85 \\
\hline
\end{tabular}


Existen 164 artículos indexados en revistas, de estos, 75 son los más referenciados en las Bases de Datos Científicas (tabla 7). La revista indexada en donde se han publicado más artículos referentes al tema de estudio es "Journal of Theorical and Applied Information Technology" con 12 publicaciones, seguida de "Lecture Notes in Business Information Processing” con 11 publicaciones. Esta tabla facilitará a los investigadores interesados en la línea de investigación para la publicación de futuros trabajos científicos.

Tabla 7. Artículos indexados en revistas más referenciados respecto a Gobierno de TI en Universidades

\begin{tabular}{|c|c|c|}
\hline \multicolumn{3}{|c|}{$\begin{array}{l}\text { REVISTAS INDEXADAS MAS REFERENCIADAS EN BASES DE } \\
\text { DATOS CIENTÍFICAS }\end{array}$} \\
\hline Nro. & REVISTAS & REFERENCIADOS \\
\hline 1 & $\begin{array}{l}\text { Journal of Theoretical and Ápplied } \\
\text { Information Technology }\end{array}$ & 12 \\
\hline 2 & $\begin{array}{l}\text { Lecture Notes in Business Information } \\
\text { Processing }\end{array}$ & 11 \\
\hline 3 & Journal of Global Information Management & 6 \\
\hline 4 & $\begin{array}{l}\text { Information Management \& Computer } \\
\text { Security }\end{array}$ & 5 \\
\hline 5 & $\begin{array}{l}\text { Advances in Intelligent Systems and } \\
\text { Computing }\end{array}$ & 4 \\
\hline 6 & $\begin{array}{l}\text { Communications in Computer and } \\
\text { Information Science }\end{array}$ & 4 \\
\hline 7 & Decision Support Systems & 3 \\
\hline 8 & Electronic Commerce Research & 3 \\
\hline 9 & Journal of Strategic Information Systems & 3 \\
\hline 10 & $\begin{array}{l}\text { International Journal of Information Systems } \\
\text { in the Service Sector }\end{array}$ & 3 \\
\hline 11 & $\begin{array}{l}\text { International Journal of ITiBusiness } \\
\text { Álignment and Governance }\end{array}$ & 3 \\
\hline 12 & Information Systems Management & 3 \\
\hline 13 & $\begin{array}{l}\text { Information Management and Computer } \\
\text { Security }\end{array}$ & 3 \\
\hline 14 & Procedia Computer Science & 2 \\
\hline 15 & $\begin{array}{l}\text { International Journal of Corporate } \\
\text { Governance }\end{array}$ & 2 \\
\hline 16 & Intelligent Decision Technologies & 2 \\
\hline 17 & Intelligent Systems Reference Library & 2 \\
\hline 18 & $\begin{array}{l}\text { International Journal of Computer and } \\
\text { Information Technology }\end{array}$ & 2 \\
\hline 19 & Information Systems Control Journal & 2 \\
\hline & TOTAL & 75 \\
\hline
\end{tabular}

De las 386 publicaciones encontradas, existen 32 de asociaciones internacionales de alto prestigio (tabla 8). Se observa que la Asociación en donde se han seleccionado más documentos referentes al tema de estudio es CRUE-TIC (Comisión Sectorial TIC de la Conferencia de Rectores de las Universidades Españolas - tic.crue.org) con 13 publicaciones. Ello es debido al sesgo de los autores y a la fuerte implicación con dicha asociación. Hay que tener en cuenta que 
la búsqueda de trabajos de investigación en asociaciones no ha seguido una metodología sistemática, si no que se ha basado fundamentalmente en la experiencia de los autores. Le siguen otras asociaciones con un menor número de publicaciones referentes a Gobierno de TI en Universidades pero que están igualmente preocupadas en el tema, tales como EUNIS (European University Information Systems - www.eunis.org), ISACA (www.isaca.org) e ITGI (www.isaca.org/About-ISACA/IT-Governance-Institute), entre otras.

\section{Tabla 8. Asociaciones Internacionales más referenciadas respecto a Gobierno de TI en Universidades}

\begin{tabular}{|c|l|c|}
\hline \multicolumn{3}{|c|}{$\begin{array}{c}\text { ASOCIACIONES INTERNACIONALES CON MÁS CONTRIBUCIONES DE } \\
\text { PUBLICACIONES DE LITERATURA NO CONVENCIONAL }\end{array}$} \\
\hline Nro. & \multicolumn{1}{|c|}{ ASOCIACIONES INTERNACIONALES } & REFERENCIADOS \\
\hline 1 & CRUE-TIC & 13 \\
\hline 2 & EUNIS & 4 \\
\hline 3 & IEEE & 2 \\
\hline 4 & ISACA & 6 \\
\hline 5 & ISO/IEC & 3 \\
\hline 6 & IT Governance Institute & 4 \\
\hline & \multicolumn{2}{r|}{ TOTAL } \\
\hline
\end{tabular}

\section{Discusiones}

De acuerdo a la investigación realizada, el Gobierno de TI en las Universidades es de interés de la comunidad de investigadores. En el periodo tomado para la investigación, del 2000 al 2017, se han encontrado 386 publicaciones donde se nota claramente que el interés va creciendo desde 3 publicaciones en el 2000 hasta 53 y 47 en el 2014 y 2015 respectivamente. Esto va de la mano del incremento de servicios tecnológicos en las universidades y que estos servicios están apuntando a los objetivos estratégicos de las universidades que están relacionados con la academia, la investigación y la vinculación con la comunidad.

El mayor número de artículos encontrados está en el área de las Ciencias de Computación con el 41\%. Si a esto se le añade las áreas de conocimiento relacionadas como la Ingeniería y las Tecnologías de la Información, se llegaría al 72\%, dando un 28\% para otras áreas diferentes a TI que tienen interés en el tema. Las áreas interesadas son de Administración, Economía, Finanzas y Ciencias de la Decisión que tienen clara influencia en los planes estratégicos de cualquier empresa y en particular de las universidades. Esto tiene mucha importancia desde el punto de vista de generar trabajos multidisciplinarios que permitan una buena administración de los recursos, aplicaciones, infraestructura e información en las universidades que permitan a las mismas un diferenciador estratégico para su acreditación y crecimiento.

La tabla 2 muestra que el $82 \%$ de las publicaciones está en las bases científicas. El porcentaje de contribución de las bases de datos no convencionales es del 18\%, un porcentaje considerable, sobre todo desde el aporte científico y práctico de los mismos.

Las Figuras 2, 3 y 4 muestran el interés de la comunidad científica por la investigación de Gobierno de TI en las Universidades. La mayoría de las publicaciones de base de datos 
científicas son de áreas relacionadas a Tecnología de la Información (Ciencias de Computación, Ingeniería y Tecnologías de la Información), de la misma manera se presenta en la literatura no convencional. De las 316 publicaciones de bases científicas el 69\% es de las áreas de tecnología y de las 70 publicaciones de la literatura no convencional el $87 \%$ es de las áreas de tecnología de la información, siendo una participación mayor en la literatura no convencional. Esto se debe a que existe un importante número de asociaciones internacionales, de estándares y de buenas prácticas tecnológicas, con mucho prestigio contribuyendo al Gobierno de las TI con el fin de alinear los objetivos estratégicos de las empresas a los servicios tecnológicos. Se muestra que en la literatura de asociaciones internacionales existe un gran interés sobre todo en el área de Tecnología de la Información. Estas asociaciones son CRUE TIC, EUNIS, ITIL (OGC), COBIT (ISACA), ISO 20000, ISO 38500.

La mayor parte de publicaciones son en idioma ingles (el 88\%), aunque existe un $12 \%$ en español, esto debido a que el inglés es el idioma más usado para la publicación en las bases científicas. Sin embargo, también hay una contribución importante en idioma español con gran importancia en su contribución científica.

Se presenta en la tabla 6 una interesante información sobre los congresos que más referencian la investigación sobre Gobierno de TI en las universidades que dan una referencia para que los investigadores en este tema puedan escoger donde publicar sus artículos. La IEEE encabeza el top 10 en cuanto a congresos y en cuanto a revistas indexadas la primera posición la ocupa el Journal of Theoretical an Applied Information Technology.

\section{Conclusiones}

En este apartado final vamos a comentar las principales conclusiones que hemos extraído de este trabajo de investigación:

La comunidad científica tiene un interés real y progresivo sobre la línea de investigación de Gobierno de TI en general, y en particular se han realizado trabajos específicos para las Universidades, donde se debe seguir trabajando.

La mayor cantidad de publicaciones son del área de Tecnología de la Información, pero hay un interés de las áreas de Negocio, Finanzas, Economía, lo que permite concluir que es posible trabajar en el tema con la participación multidisciplinaria de las áreas. Esto beneficiaría a la alineación de TI con la estrategia de la Universidades para un mejor Gobierno Corporativo.

La mayor parte de publicaciones son artículos en revistas indexadas lo que nos lleva a concluir que existe un interés sólido en la investigación formal, más sin embargo también las bases de datos no convencionales aportan en el tema.

El interés de los investigadores ha ido creciendo en el transcurso de los años desde el año 2000 hasta la presente fecha, lo que indica que se seguirá trabajando en esta línea de investigación.

Se pudo comprobar que existen grupos científicos que han desarrollado estándares y buenas prácticas alrededor del Gobierno de TI. Cabe señalar que tiene gran influencia y prestigio 
en la comunidad y los negocios. Estas asociaciones son CRUE TIC, EUNIS, ITIL (OGC), COBIT (ISACA), ISO 20000, ISO 38500.

\section{Bibliografía}

Sánchez-Meca, J. (2010). Cómo Realizar Una Revisión Sistemática Y Un Meta-Análisis (Vol. 38). (U. D. Oviedo, Ed.) Murcia, España: Aula Abierta.

Ferreras, M. F. (2016). Visibilidad E Impacto De La Literatura Gris Científica En Repositorios Institucionales De Acceso Abierto. Estudio De Caso Bibliométrico Del Repositorio Gredos De La Universidad De Salamanca. Salamanca, España: Universidad De Salamanca.

Literature. T. I. (1997). Perspectives on the Design and Transfer of Scientific and Technical Information. Amsterdam, Netherlands.

Fernández, A. M. (2009). Análisis, Planificación Y Gobierno De Las Tecnologías De La Información En Las Universidades. Almería: Universidad De Almería.

Fernández, A. M., \& Llorens, F. L. (2011). Fernández Martínez, A., Y Llorens Largo, F. (2011). Gobierno De Las TI Para Universidades (1a Ed.). Almería, España: Conferencia De Rectores De Las Universidades Españolas (CRUE). Almería: CRUE.

Uceda, J. A., Barro, S. A., Llorens, F. L., \& Franco, J. T. (2010). universitic 2010: Evolución De Las TIC En El Sistema Universitario Español 2006-2010. Madrid: Conferencia De Rectores De Las Universidades Españolas (CRUE).

Fernández, A. M., Llorens, F. L., \& Hontorio, E. H. (2015). universitic: IT'S Survey in Spanish and Latin American Universities EUNIS Research and Analysis Initiative - ERAI. . Madrid: European University Information Systems (EUNIS).

Bin-Abbas, H., \& Bakry, S. H. (2014). Assessment Of IT Governance In Organizations: A Simple Integrated Approach. Computers In Human Behavior, 261, 267. 